\title{
A Comparison of Girls' and Boys' Aggressive-Disruptive Behavior Trajectories Across Elementary School: Prediction to Young Adult Antisocial Outcomes
}

\author{
Cindy M. Schaeffer \\ University of Maryland Baltimore County
}

Nicholas Ialongo

Johns Hopkins Bloomberg School of Public Health

Scott Hubbard

Johns Hopkins Bloomberg School of Public Health
Hanno Petras

University of Maryland

Katherine E. Masyn

University of California, Davis

\begin{abstract}
Multiple group analysis and general growth mixture modeling was used to determine whether aggressive-disruptive behavior trajectories during elementary school, and their association with young adulthood antisocial outcomes, vary by gender. Participants were assessed longitudinally beginning at age 6 as part of an evaluation of 2 school-based preventive programs. Two analogous trajectories were found for girls and boys: chronic high aggression-disruption (CHAD) and stable low aggressiondisruption (LAD). A 3rd class of low moderate aggression-disruption (LMAD) for girls and increasing aggression-disruption (IAD) for boys also was found. Girls and boys in analogous CHAD classes did not differ in trajectory level and course, but girls in the CHAD and LAD classes had lower rates of antisocial outcomes than boys. Girls with the LMAD trajectory differed from boys with the IAD trajectory.
\end{abstract}

Keywords: aggression, trajectories, growth mixture modeling, multiple group comparison, gender differences

There has been considerable interest in understanding divergent developmental pathways leading to antisocial outcomes in adolescence and young adulthood, in both the theoretical (e.g., Moffitt, 1993; Patterson, DeBaryshe, \& Ramsey, 1989) and empirical (e.g., Nagin \& Tremblay, 1999; Schaeffer, Petras, Ialongo, Poduska, \&

Cindy M. Schaeffer, Department of Psychology, University of Maryland-Baltimore County; Hanno Petras, Department of Criminology and Criminal Justice, University of Maryland; Nicholas Ialongo and Scott Hubbard, Department of Mental Health, Johns Hopkins Bloomberg School of Public Health; Katherine E. Masyn, Department of Human and Community Development, University of California, Davis; Jeanne Poduska and Sheppard Kellam, American Institutes for Research.

This research was supported by grants from the National Institutes of Mental Health (RO1 MH42968 [Development and Malleability from Childhood to Young Adulthood, Sheppard Kellam, Principal Investigator] and T-32 MH18834 [Training Program in Prevention Research, Nicholas Ialongo, Principal Investigator]) and the Centers for Disease Control and Prevention (R49/CCR318627-03). We thank the Baltimore City Public Schools for their continuing collaborative efforts and the parents, children, teachers, principals, and school psychologists/social workers who participated. We also express our appreciation to Sharon Lambert, who made significant contributions to the editing of this article, and to Bengt Muthén, who provided valuable consultation on statistical modeling techniques.

Correspondence concerning this article should be addressed to Cindy M. Schaeffer, Department of Psychology, 1000 Hilltop Circle, University of Maryland Baltimore County, Baltimore, MD 21250. E-mail: cschaeff@umbc.edu
Kellam, 2003) literatures. From this work, it is clear that a pattern of chronic aggressive behavior in childhood is a significant risk factor for antisocial behavior in later life, at least for boys. However, the empirical literature has focused primarily on the development of boys' aggression and has given little attention to the role of early aggression and other disruptive behaviors in the development of girls' later antisocial behavior (Keenan \& Shaw, 1997; Silverthorn \& Frick, 1999). Although some empirical studies examining pathways of antisocial behavior development among girls are starting to emerge in the literature (e.g., Broidy et al., 2003 Cote, Zoccolillo, Tremblay, Nagin, \& Vitaro, 2001), research regarding gender differences in these pathways is limited. The primary goals of the present study were to examine developmental trajectories of girls' antisocial behavior over the elementary school years and to empirically compare the initial level, course, and association with later adult antisocial behavior of girls' trajectories to those of boys.

\section{Models of Antisocial Behavior Development}

Regarding the development of antisocial behavior in boys, there is agreement in the research literature that there are at least two qualitatively different developmental pathways to serious criminal involvement (Bierman et al., 2004). The most serious of these is the "early-starting" or "life-course-persistent" pathway described in the developmental models of Patterson et al. (1989) and Moffitt 
(1993). ${ }^{1}$ This pathway is characterized by high levels of physical aggression beginning in early childhood and continuing throughout development, resulting in criminal and violent behavior in adolescence and adulthood. Youth on this pathway have a variety of early and ongoing risk factors, including neurological deficits (e.g., attention problems) and social problems (e.g., coercive parenting, peer rejection, and school failure). These models imply that this pathway and the mechanisms leading to antisocial outcomes are gender invariant, an assumption with some empirical support (e.g., Fergusson \& Horwood, 2002; Webster-Stratton, 1996).

A comprehensive theory of the development of antisocial behavior that is specific to girls does not yet exist. Some researchers have questioned the applicability of the early-starter model to girls' development, given that only very small numbers of girls exhibit high rates of aggression in early childhood (Moffitt, Caspi, Rutter, \& Silva, 2001; Silverthorn \& Frick, 1999). Other researchers have argued that an early-starter pathway can be detected for girls if a broader definition of antisocial behaviors is used, one that includes oppositional (e.g., arguing) and disruptive (e.g., tantruming) behavior in addition to physical aggression, and that to broaden in this way would result in more effective screening of at-risk girls (Bierman et al., 2004). Assuming that an early-starter pathway does exist for girls, another question pertains to whether there is meaningful heterogeneity within this pathway (i.e., more than one early-starter pattern), as is becoming increasingly evident in studies of boys (e.g., Schaeffer et al., 2003).

\section{Longitudinal Studies of Individual Differences in Antisocial Behavior Development Among Girls}

Growth modeling techniques are an effective method of detecting developmental patterns in longitudinal data (Muthén, 2004) and of providing tests of developmental theory. Many studies have used growth modeling techniques to examine early-starter pathways among boys (e.g., Nagin \& Tremblay, 1999; Shaw, Gilliom, Ingoldsby, \& Nagin, 2003). However, only two studies have done so using samples of girls, each focusing on different aspects of early antisocial behavior. Broidy et al. (2003) examined longitudinal patterns of teacher-reported physical aggression (e.g., fights with others, bullies other children) among girls over the course of elementary school using four epidemiological samples from New Zealand, Canada, and the United States. A distinct subgroup of girls (3\%-14\% of the sample) displaying an early-starter pattern was found in three of the four samples (including the sample from the United States). Although not explicitly tested, girls with the early-starter pattern appeared to have lower absolute levels of aggression (i.e., lower mean scores) over time than did their counterpart male early starters. Also, the link between the earlystarter pattern and later violent and nonviolent delinquent behavior was less consistent for girls across samples than it was for boys (i.e., only 6 of 9 analyses that examined this association for girls were significant, as compared with 9 of 10 analyses for boys).

Using a broader definition of antisocial behavior, Cote et al. (2001) examined the development of teacher-rated aggressive (e.g., fights with other children, bullies other children) and disruptive (e.g., disobedient, doesn't share) behavior from ages 6 to 12 in an epidemiological sample of Canadian girls and found two earlystarter patterns, a high and a medium-high group. Both of these groups were at increased risk for a diagnosis of later conduct disorder relative to nondisruptive girls. These findings lend support to Bierman et al.'s (2004) suggestion that early-starter patterns among girls may be best captured by the inclusion of both physically aggressive and disruptive/oppositional behavior. These findings also suggest that a broader definition of antisocial behavior may lead to the detection of more than one early-starter pattern among girls.

\section{Present Study Rationale and Goals}

The present study addresses gaps in our knowledge regarding potential similarities and differences between girls and boys in the developmental course of early antisocial behavior and its association to antisocial outcomes in adulthood. Building on existing theories of antisocial behavior development (Moffitt, 1993; Patterson et al., 1989) and available empirical evidence (Broidy et al., 2003; Cote et al., 2001), we tested whether one or more earlystarter patterns could be identified in a sample of primarily African American elementary school girls and whether these patterns were comparable with those of boys. Given the inconclusive findings of Broidy et al. (2003) regarding gender differences in the association between early trajectories of antisocial behavior and later antisocial outcomes, we also compared girls and boys on the prevalence of outcomes associated with these developmental trajectories. In accord with the recommendation of Bierman et al. (2004) and the findings of Cote et al. (2001), we used a broad definition of early antisocial behavior that includes both physically aggressive and disruptive behaviors.

The present study is an extension of earlier work examining trajectories of aggressive-disruptive behavior among boys from the same data set (Petras et al., 2004; Schaeffer et al., 2003). Given our use of a broad measure of antisocial behavior, we based our hypotheses regarding girls' trajectory patterns on the findings of Cote et al. (2001). Specifically, we anticipated three distinct groups of girls: a chronic high early-starter group whose aggressive-disruptive behavior would be elevated on entry to first grade and remain so throughout the elementary school years; a second early-starter group showing a pattern of moderate aggressive-disruptive behavior over time; and a group of girls whose aggressive-disruptive behavior would begin and remain consistently low.

Previous studies did not explicitly test for gender differences in early-starter patterns of antisocial behavior. As noted, although girls and boys have been shown to have analogous patterns of aggression over time (Broidy et al., 2003), the absolute levels of aggression appeared to be lower for girls. In addition, trajectory memberships were not as consistently associated with later antisocial outcomes for girls as they were for boys. Without specific empirical tests of these apparent gender differences, these findings are inconclusive. In the present study, we expected that, although the hypothesized trajectories for girls would be analogous to those found for boys, the absolute level of aggression-disruption over time within these trajectories would be lower than those found for boys (based on the results of Broidy et al., 2003). Also consistent

\footnotetext{
${ }^{1}$ The other pathway proposed in these models, "late starters" or "adolescent-limited offenders," involve youth who also are at risk for later antisocial behavior; this pathway is not a focus of the present study.
} 
with the existing research, we expected that, although girls with chronic high or moderate aggression-disruption would be more at risk for antisocial outcomes than would nonaggressive-disruptive girls, their level of risk would be lower than that of boys with analogous early-starter patterns.

The present study used the newest generation of latent growth modeling techniques, general growth mixture modeling (GGMM; Muthén, 2004), to classify systematic variation in the nature and course of aggressive-disruptive behavior and to assess whether these classifications are associated with later antisocial outcomes. Consistent with Muthén (2004), we incorporated a range of theoretically derived covariates to better specify classes in our models. ${ }^{2}$ On the basis of Moffitt's (1993) theory that neuropsychological deficits such as attention problems are higher among youth with chronic high aggression, we included teacher-rated concentration problems as a covariate. Because Patterson et al. (1989) suggested that rejection from mainstream peers and poor school performance frequently co-occur with early antisocial behavior, we also controlled for peer rejection and reading achievement. Finally, given the role of economic deprivation in youth involvement in antisocial behavior and the fact that in urban environments African American youth are more likely than European American youth to experience poverty (Ingoldsby \& Shaw, 2002), we also controlled for the effects of race and free lunch status (a proxy variable for family poverty) in all analyses.

\section{Method}

\section{Participants}

Participants included 664 girls and 675 boys who were first assessed at age 6 as part of an evaluation of two school-based, universal preventive interventions targeting early learning and aggression in first and second grade (Dolan et al., 1993) in 19 Baltimore, MD, public schools. These youth were members of the control group within the evaluation design. The schools were drawn from five geographic areas within the eastern half of the city, defined by census tract data and vital statistics obtained from the Baltimore City Planning Office.

A total of 1,339 control participants were originally available within the 19 participating schools in first grade. One hundred fifty-eight ( 80 girls, 78 boys) control youth did not have a teacher rating from the fall of first grade, and an additional 44 were missing other covariate information; consequently, they were not included in the analyses for this article. ${ }^{3}$ The resulting sample of 1,137 youth consisted of 558 girls $(71.3 \%$ African American, 28.7\% European American) and 579 boys (61.3\% African American and $38.7 \%$ European American). At entrance into first grade, the average age was 6 years (girls $=6.2$ years $[S D=0.41]$, boys $=6.3$ years $[S D=0.47])$. Approximately half of the youth $(52.7 \%$ of girls and $52 \%$ of boys) received free or reduced-price school lunch, a proxy for low family income. There were no differences in terms of race, age, or achievement test scores between the 202 youth with missing data and the 1,137 youth with baseline teacher and covariate data.

Of the 1,137 control participants with a teacher rating from the fall of first grade, 53 (14 girls and 39 boys) refused to participate in the follow-up for ages 19-20, 19 (4 girls and 15 boys) had died (as confirmed by a search of the National Death Index and/or an immediate family member), and 197 young adults (74 girls, 123 boys) either failed to respond to repeated requests for an interview or were unable to be located during the fielding period. Thus, of those youth contributing baseline data who were alive at the time of follow-up, $84.1 \%$ of girls $(n=466)$ and $71.3 \%$ of boys $(n=$ 402) contributed data at ages $19-20 .^{4}$

\section{Assessment Design}

Data for this study were gathered in the fall and spring of first grade, the spring of second through fifth grades, and at the follow-up assessment for ages 19-20. The data gathered in the first-grade assessments included teacher reports of child aggressive-disruptive behavior, attentionconcentration problems, and peer rejection. Data on free-lunch eligibility and race also were collected in first grade. Teacher reports of child aggressive-disruptive behavior were collected annually or semiannually in Grades 1-5. At ages 19-20, a follow-up structured clinical interview was used to ascertain whether the participant met criteria for antisocial personality disorder, and adult adjudication records were obtained.

\section{Measures}

Teacher Observation of Classroom Adaptation-Revised (TOCA-R; Werthamer-Larsson, Kellam, \& Wheeler, 1991). Teacher ratings of aggressive-disruptive behavior, attention-concentration problems, and peer rejection were obtained in the fall and spring of first grades using the TOCA-R. Thereafter, teacher ratings of aggressive-disruptive behavior using the TOCA-R were collected annually in the spring of Grades 2-5. Thus, although the first-grade variables were rated by the same teacher, each subsequent year a different teacher provided ratings.

The TOCA-R is a structured interview with the teacher, which is administered by a trained assessor. Teachers respond to 36 items pertaining to the child's adaptation to classroom task demands over the previous 3 weeks. Adaptation is rated by teachers on a 6-point frequency scale ranging from 1 (almost never) to 6 (almost always). The aggressive-disruptive behaviors subscale includes the following 10 items: breaks rules, harms others and property, breaks things, takes others' property, fights, lies, has trouble accepting authority, yells at others, is stubborn, and teases classmates. Factor-analytic work has determined the factor structure of this subscale to be gender invariant (Greenbaum et al., 2000). On average, across time points, girls' scores on this scale were 0.26 of a standard deviation lower than the scores of boys in this sample; one-way analyses of variance indicated that boys' scores on this subscale were significantly higher $(p<.001)$ at all time points. The coefficient alphas for the aggressive-disruptive behaviors subscale ranged from .92 to .94 over Grades 1 through 5 or ages $8-11 .^{5}$

Regarding the TOCA-R's attention-concentration subscale, Werthamer-Larsson et al. (1991) reported alphas of .91 and .83 in first graders. The subscale items are as follows: completes assignments, con-

\footnotetext{
${ }^{2}$ Muthén (2004) has suggested that auxiliary information in the form of antecedents (covariates) of class membership and growth factors should be included to correctly specify models, find the proper number of classes, and correctly estimate class memberships and class proportions. If classes are not statistically different with respect to covariates that, according to theory, should distinguish classes, crucial support for the model is absent. Thus, the covariates and distal outcomes in the present study provide evidence that the derived classes represent meaningful population heterogeneity.

${ }^{3}$ These cases would have been excluded by the Mplus software used in our analyses. The algorithm excludes cases that are missing covariates. In all cases, youth missing teacher-rated aggression in the fall of first grade also were missing data on covariates measured at that same time point.

${ }^{4}$ Because full information maximum likelihood estimation was used in all analyses (under the assumption that the data are missing at random), parameter estimates were based on all available time points for a given case. Thus, the sample size for all analyses was 1,137 .

${ }^{5}$ Full descriptive information regarding the TOCA-R (e.g., means, standard deviations) in this sample are available from Cindy M. Schaeffer upon request.
} 
centrates, poor effort, works well alone, pays attention, learns up to ability, eager to learn, works hard, and stays on task. In terms of concurrent validity, each single unit of increase in teacher-rated attentionconcentration problems was associated with a twofold increase in risk of teacher perception for the need for medication for such problems. Teacher ratings of peer rejection were based on a single item, "rejected by classmates," with 1 indicating total acceptance and 6 indicating total rejection. The 4-month intraclass correlation coefficient for this item was .74, and the item correlated significantly with peer nominations (not reported in this study) for the questions "Which kids don't you like?" $(r=.43)$ and "Which kids are your best friends?" $(r=-.58)$

Reading achievement. Our measure of reading achievement comes from the California Achievement Test (CAT; Forms E \& F), which was administered in the fall of first grade. The CAT represents one of the most frequently used standardized achievement batteries (Wardrop, 1989). The CAT was standardized on a nationally representative sample of 300,000 children. Internal consistency coefficients for virtually all of the subscales including the reading subscale exceed .90 . Alternate form reliability coefficients are in the .80 range (Wardrop, 1989).

Eligibility for free lunch. Eligibility for a free school lunch on entry into first grade was chosen as a proxy for family income in the present study. Conceptually, eligibility for free lunch is likely to be a proxy variable for a range of economic and other stressors operating at the family and neighborhood levels. Previous research has demonstrated that freelunch eligibility correlates highly with family income and other traditional measures of socioeconomic status (Ensminger et al., 2000). Eligibility was treated as a binary variable (i.e., $0=$ not eligible, $1=$ eligible). In the present study, free-lunch status had a strong negative correlation with parent education status.

Antisocial personality disorder (ASPD) diagnosis. As part of a larger telephone interview at ages 19-20, we used a scale developed by Turner and Gil (2002) to determine whether the participant met Diagnostic and Statistical Manual of Mental Disorders (4th ed.; DSM-IV; American Psychiatric Association, 1994) criteria for ASPD. The questions constituting the scale were keyed to $D S M-I V$ criteria, and the diagnoses were derived in accord with those criteria. To reduce the likelihood of socially desirable responses, we asked participants to maintain their own count of "yes" responses as opposed to responding "yes" or "no" to the interviewer's questions. To ensure against the respondents losing track of the count, we asked them to have a pencil and sheet of paper available to mark down the number of "yes" responses. In addition, the questions were divided into three sections and a count of "yes" responses was obtained by the interviewer at the end of each section. Telephone interviews as a way of gathering diagnostic information have been shown to be a valid means of measuring a range of psychiatric disorders in many studies (e.g., Kobak et al., 1997; Rohde, Lewinsohn, \& Seeley, 1997; Slutske et al., 1998). In terms of concurrent validity in the present study, relative to those who did not meet criteria for ASPD, participants with ASPD were significantly more likely to have a criminal record (girls' odds ratio $[\mathrm{OR}]=4.91,95 \%$ confidence interval $[\mathrm{CI}]=2.76,8.70$; boys' $\mathrm{OR}=3.40,95 \% \mathrm{CI}=2.38$, 4.83).

Adult offense and incarceration records. Adult offense and incarceration records were obtained at the time of the young adult follow-up interview (i.e., at age 20). Adjudication for a violent (e.g., assault, rape) crime constituted our violent offense variable; these data were obtained from local records in Baltimore City and the five municipalities surrounding the city. Incarceration for an offense classified as a felony in the Uniform Crime Reports system (i.e., murder, nonnegligent manslaughter, forcible rape, robbery, aggravated assault, burglary, larceny/theft, and motor vehicle theft) was used as an indicator of serious antisocial behavior as an adult and is hereinafter referred to as incarceration; these data were obtained from the Maryland Department of Public Safety and Correctional Services. Both offense and incarceration data were treated as binary variables (i.e., absence $=0$, presence $=1$ ).

\section{Statistical Methodology}

GGMM. We used GGMM (Muthén, 2004; Muthén \& Shedden, 1999), with the Mplus Version 3.12 statistical software package (Muthén \& Muthén, 2004), to identify patterns of growth in aggressive-disruptive behavior over time. Like traditional growth modeling techniques, GGMM estimates growth parameters associated with latent variables (growth factors) manifested by repeated measures of a univariate outcome over time (multiple indicators). GGMM attempts to capture sample heterogeneity by representing the population distribution by two or more distinct classes of developmental trajectories with random variability around the mean trajectories within each class. Although it is difficult empirically to distinguish between data that have been drawn from a single nonnormal population distribution and data from a mixture of normal subpopulations (Bauer \& Curran, 2003), the hypothesis of different pathways in aggressive-disruptive behavior development is supported when the data are better fit with a growth mixture model of two or more latent classes than with a traditional growth model. ${ }^{6}$

The observed outcomes consisted of teacher-rated classroom aggression-disruption measured at six time points: fall and spring of first grade, and spring of second through fifth grades. Time was treated as a fixed parameter in the models. The time points were fixed incrementally based on the spacing between assessment sessions (fall of first grade fixed at 0 , spring of first grade fixed at 0.5 , spring of second grade fixed at 1.5 , etc.). In all models, standard errors were adjusted by the sandwich estimator to account for the clustered sampling design (i.e., students within classrooms within schools). All analyses used automated multiple starting values in the optimization to reduce the risk that solutions represent local rather than global optima.

Multiple group analysis within GGMM. Multiple group analysis was used to test for gender differences on baseline levels of aggressivedisruptive behavior, course of this behavior over time, and the classspecific prevalence of the distal outcomes (i.e., ASPD, violent arrest, and incarceration). The multiple groups approach is preferable to simply treating gender as a covariate in the models (i.e., the Multiple Indicator Multiple Causes [MIMIC] approach; Jöreskog \& Goldberger, 1975), which would impose many equalities between genders that may not be valid (e.g., number of trajectory classes, structure of classes, within-class variability, residual variances, effects of covariates, and association between class membership and distal outcomes).

For the multiple groups analysis, we first ran the separate GGMM models for girls and boys to determine the number of trajectory classes, the shape of growth within aggressive-disruptive behavior trajectories, and the prevalences of the distal outcomes by class. The resulting two models informed our base multiple group model, which assumed nonequivalent parameters across genders by trajectory class. Then, we investigated if any of the parameters could be constrained to be equal across the two gender groups. We limited our comparisons to those parameters of interest, namely, the class specific: starting points in aggressive-disruptive behavior (i.e., intercept); courses of aggression-disruption over time (i.e., slope/ quadratic slope); and prevalences of distal outcomes.

\footnotetext{
${ }^{6}$ In a previous report (Schaeffer et al., 2003) we raised the possibility that special statistical methods may be needed to account for large numbers of girls having very low scores (Olsen \& Schaefer, 2001). We applied a new method, censored normal growth mixture modeling, to control for the truncated distribution of aggressive-disruptive behavior scores among girls in our sample, but there was no indication that it improved the fit or interpretability of the models for girls, leading us to choose standard GGMM for all analyses.
} 


\section{Missing Data}

The Mplus software uses a full information maximum likelihood estimation under the assumption that the data are missing at random (MAR; Arbuckle, 1996; Little, 1995), which is a widely accepted way of handling missing data (Muthén \& Shedden, 1999; Schafer \& Graham, 2002). Overall, $75 \%$ of the participants had at least four of the six assessment time points from Grades $1-5$. The minimum covariance coverage recommended for reliable model convergence is .10 (Muthén \& Muthén, 2004). In this study, coverage ranged from .47 to .89 .

\section{Results}

\section{Descriptive Statistics}

The overall prevalence of the antisocial distal outcomes in the sample were as follows: For girls, 6.0\% had a diagnosis of ASPD, $7.5 \%$ were arrested for a violent crime, and $0.4 \%$ were incarcerated; for boys, $21.4 \%$ had a diagnosis of ASPD, $14.8 \%$ were arrested for a violent crime, and $13.5 \%$ were incarcerated.

\section{Growth Models and Trajectory Classes}

Before we tested for gender differences in aggressivedisruptive behavior trajectories, it first was necessary to determine the nature of growth in these behaviors for girls and boys in separate models. Because the models for boys are reported elsewhere (Petras et al., 2004), only the models for girls are presented. Consistent with Muthén et al. (2002), information criteria were obtained for one-, two-, three-, and four-class solutions. In these models, covariates were allowed to influence latent growth parameters (i.e., intercept, slope, quadratic slope) as well as class membership. In an effort to ensure that trajectories were based solely on the heterogeneity of aggressive-disruptive behavior and not also on the heterogeneity of our control variables, we constrained the effects of covariates on growth parameters (i.e., intercept, slope, quadratic slope) to be the same across classes. We also made the assumption that any associations between the starting point of aggression-disruption (i.e., the intercept) and its course over time (i.e., linear and quadratic growth) should not be controlled for and, instead, should be captured through differential trajectory class memberships (e.g., that variation in the association between intercepts and slopes represent different developmental pathways); accordingly, we set the covariances between intercept/slope, intercept/quadratic slope, and slope/quadratic slope factors (which were small, not significantly different from zero) to zero before deriving classes.

Because models with different numbers of latent classes are not nested, the Bayesian Information Criterion (BIC; Schwartz, 1978) was used to select the best model. The BIC suggested that a three-class solution was optimal $(\mathrm{BIC}=5,081.94)$. On the basis of the findings of Muthén et al. (2002) and Petras et al. (2004), we assumed that girls in the nonaggressive-nondisruptive class would show less variability in aggressive-disruptive behavior than girls in other trajectory classes; accordingly, we allowed for residual variances to be freely estimated for that class. Also, given our assumption that girls in the nonaggressive-nondisruptive class would not show growth in aggression-disruption over time, we fixed the means of the linear and quadratic growth factors (which were small and nonsignificant) to zero. These modifications resulted in better fit relative to the unmodified three-class model
$(\mathrm{BIC}=4,511.73)$ and showed good precision in class membership estimation (entropy $=.914$ ). In a final step, three distal outcomes (ASPD, violent arrest, and incarceration) were regressed upon the class membership.

The girls' model identified three distinct trajectories of aggressive behavior: a chronic high aggressive-disruptive behavior (CHAD) trajectory, consisting of those girls ( $n=49$, or $9 \%$ of the sample of girls) whose aggression-disruption started high in first grade and declined through third grade, where it appeared to stabilize; a low moderate aggressive-disruptive behavior (LMAD) trajectory, consisting of girls $(n=373$, or $67 \%)$ whose aggression-disruption remained at a low moderate level between first and fifth grades; and a low aggressive-disruptive behavior (LAD) trajectory, consisting of girls $(n=135$, or $24 \%$ ) with consistently low levels of the behavior over time. Three distal outcomes (ASPD, violent arrest, and incarceration) were regressed upon the class membership variable (see Table 1 for prevalences of outcomes by class). Relative to girls with LAD, membership in the CHAD class was associated with significantly increased risk for ASPD $(\mathrm{OR}=5.81 ; 95 \% \mathrm{CI}=1.94,17.40)$ and arrest for a violent offense $(\mathrm{OR}=15.51 ; 95 \% \mathrm{CI}=3.22,74.79)$, but not for incarceration $(\mathrm{OR}=1)$. Girls with a pattern of LMAD were not at significantly increased risk for ASPD or incarceration, but they were more likely to have a violent arrest $(\mathrm{OR}=6.65 ; 95 \% \mathrm{CI}=$ 1.61, 27.40). Girls with CHAD also had increased risk for ASPD $(\mathrm{OR}=3.99 ; 95 \% \mathrm{CI}=1.62,9.84)$ and violent arrest $(\mathrm{OR}=2.33$ $95 \% \mathrm{CI}=1.01,5.37)$ relative to girls with $\mathrm{LMAD}^{7}$

As described in Petras et al. (2004), three distinct aggressivedisruptive behavior trajectories were identified for boys: a CHAD (15\% of boys) trajectory; an increasing aggressive-disruptive behavior (IAD, $52 \%$ of boys) trajectory; and a LAD (33\% of boys) trajectory. Trajectory classes for girls and boys, adjusted for the effects of covariates, are shown in Figure 1.

In our examination of the trajectories derived for girls and boys, it appeared that the CHAD and LAD trajectories were analogous to one another (i.e., they showed similar growth parameter estimates, similar class prevalences, and similar risk for a distal outcome) and thus should be tested for class-specific gender differences. It was more difficult to judge whether the girls' LMAD and boys' IAD trajectories were analogous. On the one hand, their growth parameter estimates and prevalences of distal outcomes appeared to be quite different from one another. On the other hand, both groups displayed significantly higher risk for an antisocial outcome when compared with their LAD counterparts. Ultimately, we decided to test for gender differences between LMAD and IAD groups to determine empirically the extent to which they varied on the parameters of interest, including the model-estimated risk elevations for the distal outcomes relative to their respective LAD trajectory.

\section{Empirical Tests of Gender Differences in Growth Trajectories: Multiple Group Analysis}

For the multiple group analysis, the separate models for girls and boys were combined into a single, initial model containing both genders. In this initial model, we treated class membership as

\footnotetext{
${ }^{7}$ Full results of the girls' analyses are available from Cindy M. Schaeffer upon request.
} 
Table 1

Parameter Estimates for the Multiple Group Model

\begin{tabular}{|c|c|c|c|c|c|c|}
\hline \multirow[b]{3}{*}{ Parameter } & \multicolumn{6}{|c|}{ Aggression growth estimates (and standard errors) } \\
\hline & \multicolumn{2}{|c|}{ CHAD-CHAD } & \multicolumn{2}{|c|}{ LMAD-IAD } & \multicolumn{2}{|c|}{ LAD-LAD } \\
\hline & Girls & Boys & Girls & Boys & Girls & Boys \\
\hline$\alpha_{0}$ & $3.66(0.20)$ & $3.38(0.79)$ & $1.75(0.05)$ & $1.73(0.28)$ & $1.10(0.03)_{\mathrm{a}}$ & $1.35(0.07)_{\mathrm{a}}$ \\
\hline$\alpha_{1}$ & $-0.53(0.28)$ & $-0.60(0.19)$ & $0.08(0.06)_{\mathrm{b}}$ & $0.49(0.33)_{\mathrm{b}}$ & 0.00 (Fixed) & 0.00 (Fixed) \\
\hline$\alpha_{2}$ & $0.08(0.05)$ & $0.10(0.04)$ & $-0.02(0.01)_{\mathrm{c}}$ & $-0.07(0.05)_{\mathrm{c}}$ & 0.00 (Fixed) & 0.00 (Fixed) \\
\hline $\mathrm{V}\left(\zeta_{0}\right)$ & $0.12(0.03)$ & $0.19(0.16)$ & $0.12(0.03)$ & $0.19(0.16)$ & 0.00 (Fixed) & 0.00 (Fixed) \\
\hline $\mathrm{V}\left(\zeta_{1}\right)$ & $0.02(0.00)$ & $0.02(0.02)$ & $0.02(0.00)$ & $0.02(0.02)$ & 0.00 (Fixed) & 0.00 (Fixed) \\
\hline $\mathrm{V}\left(\zeta_{2}\right)$ & 0.00 (Fixed) & 0.00 (Fixed) & 0.00 (Fixed) & 0.00 (Fixed) & 0.00 (Fixed) & 0.00 (Fixed) \\
\hline$\gamma_{0} \mid$ Concentration & $0.04(0.01)$ & $0.11(0.05)$ & $0.04(0.01)$ & $0.11(0.05)$ & $0.04(0.01)$ & $0.11(0.05)$ \\
\hline$\gamma_{0} \mid$ Reading & $0.00(0.00)$ & $0.01(0.01)$ & $0.00(0.00)$ & $0.01(0.01)$ & $0.00(0.00)$ & $0.01(0.01)$ \\
\hline$\gamma_{0} \mid$ Peer rejection & $0.03(0.03)$ & $0.18(0.12)$ & $0.03(0.03)$ & $0.18(0.12)$ & $0.03(0.03)$ & $0.18(0.12)$ \\
\hline$\gamma_{0}$ Lunch status & $-0.01(0.01)$ & $-0.01(0.12)$ & $-0.01(0.01)$ & $-0.01(0.12)$ & $-0.01(0.01)$ & $-0.01(0.12)$ \\
\hline$\gamma_{0}$ Race & $-0.01(0.01)$ & $0.21(0.06)$ & $-0.01(0.01)$ & $0.21(0.06)$ & $-0.01(0.01)$ & $0.21(0.06)$ \\
\hline$\gamma_{1} \mid$ Concentration & $-0.01(0.01)$ & $-0.02(0.02)$ & $-0.01(0.01)$ & $-0.02(0.02)$ & $-0.01(0.01)$ & $-0.02(0.02)$ \\
\hline$\gamma_{1} \mid$ Reading & $0.00(0.00)$ & $0.00(0.01)$ & $0.00(0.00)$ & $0.00(0.01)$ & $0.00(0.00)$ & $0.00(0.01)$ \\
\hline$\gamma_{1} \mid$ Peer rejection & $-0.02(0.01)$ & $-0.04(0.02)$ & $-0.02(0.01)$ & $-0.04(0.02)$ & $-0.02(0.01)$ & $-0.04(0.02)$ \\
\hline$\gamma_{1} \mid$ Lunch status & $0.03(0.01)$ & $0.03(0.04)$ & $0.03(0.01)$ & $0.03(0.04)$ & $0.03(0.01)$ & $0.03(0.04)$ \\
\hline$\gamma_{1} \mid$ Race & $0.00(0.01)$ & $-0.02(0.02)$ & $0.00(0.01)$ & $-0.02(0.02)$ & $0.00(0.01)$ & $-0.02(0.02)$ \\
\hline$\gamma_{2} \mid$ Concentration & 0.00 (Fixed) & 0.00 (Fixed) & 0.00 (Fixed) & 0.00 (Fixed) & 0.00 (Fixed) & 0.00 (Fixed) \\
\hline$\gamma_{2}$ Reading & 0.00 (Fixed) & 0.00 (Fixed) & 0.00 (Fixed) & 0.00 (Fixed) & 0.00 (Fixed) & 0.00 (Fixed) \\
\hline$\gamma_{2} \mid$ Peer rejection & 0.00 (Fixed) & 0.00 (Fixed) & 0.00 (Fixed) & 0.00 (Fixed) & 0.00 (Fixed) & 0.00 (Fixed) \\
\hline$\gamma_{2}$ Lunch status & 0.00 (Fixed) & 0.00 (Fixed) & 0.00 (Fixed) & 0.00 (Fixed) & 0.00 (Fixed) & 0.00 (Fixed) \\
\hline$\gamma_{2} \mid$ Race & 0.00 (Fixed) & 0.00 (Fixed) & 0.00 (Fixed) & 0.00 (Fixed) & 0.00 (Fixed) & 0.00 (Fixed) \\
\hline $\mathrm{V}\left(\varepsilon_{1 \mathrm{IF}}\right)$ & $0.19(0.04)$ & $0.23(0.13)$ & $0.19(0.04)$ & $0.23(0.13)$ & $0.00(0.00)$ & $0.09(0.04)$ \\
\hline $\mathrm{V}\left(\varepsilon_{1 \mathrm{~S}}\right)$ & $0.26(0.04)$ & $0.44(0.06)$ & $0.26(0.04)$ & $0.44(0.06)$ & $0.01(0.00)$ & $0.10(0.03)$ \\
\hline $\mathrm{V}\left(\varepsilon_{2 \mathrm{~S}}\right)$ & $0.40(0.05)$ & $1.11(0.14)$ & $0.40(0.05)$ & $1.11(0.14)$ & $0.31(0.13)$ & $0.17(0.05)$ \\
\hline $\mathrm{V}\left(\varepsilon_{3 \mathrm{~S}}\right)$ & $0.51(0.06)$ & $1.02(0.15)$ & $0.51(0.06)$ & $1.02(0.15)$ & $0.05(0.02)$ & $0.15(0.05)$ \\
\hline $\mathrm{V}\left(\varepsilon_{4 \mathrm{~S}}\right)$ & $0.51(0.06)$ & $0.78(0.10)$ & $0.51(0.06)$ & $0.78(0.10)$ & $0.06(0.02)$ & $0.10(0.03)$ \\
\hline $\mathrm{V}\left(\varepsilon_{5 \mathrm{~s}}\right)$ & $0.27(0.07)$ & $0.74(0.17)$ & $0.27(0.07)$ & $0.74(0.17)$ & $0.08(0.04)$ & $0.17(0.06)$ \\
\hline $\mathrm{C}\left(\varepsilon_{1 \mathrm{f}}, \varepsilon_{1 \mathrm{~S}}\right)$ & $0.00(0.00)$ & $0.07(0.03)$ & $0.00(0.00)$ & $0.07(0.03)$ & $0.00(0.00)$ & $0.07(0.03)$ \\
\hline $\mathrm{C}\left(\alpha_{0}, \alpha_{1}\right)$ & 0.00 (Fixed) & 0.00 (Fixed) & 0.00 (Fixed) & 0.00 (Fixed) & 0.00 (Fixed) & 0.00 (Fixed) \\
\hline $\mathrm{C}\left(\alpha_{0}, \alpha_{2}\right)$ & 0.00 (Fixed) & 0.00 (Fixed) & 0.00 (Fixed) & 0.00 (Fixed) & 0.00 (Fixed) & 0.00 (Fixed) \\
\hline $\mathrm{C}\left(\alpha_{1}, \alpha_{2}\right)$ & 0.00 (Fixed) & 0.00 (Fixed) & 0.00 (Fixed) & 0.00 (Fixed) & 0.00 (Fixed) & 0.00 (Fixed) \\
\hline Estimated probability of ASPD & $0.19(0.05)$ & $0.27(0.22)$ & $0.05(0.02)_{\mathrm{d}}$ & $0.27(0.09)_{\mathrm{d}}$ & $0.04(0.02)_{\mathrm{e}}$ & $0.11(0.03)_{\mathrm{e}}$ \\
\hline Estimated probability of violent offense & $0.20(0.06)$ & $0.18(0.18)$ & $0.10(0.01)_{\mathrm{f}}$ & $0.20(0.08)_{\mathrm{f}}$ & $0.02(0.01)$ & $0.05(0.02)$ \\
\hline Estimated probability of incarceration & $0.00(0.00)_{\mathrm{g}}$ & $0.16(0.15)_{\mathrm{g}}$ & $0.01(0.00)_{\mathrm{h}}$ & $0.21(0.08)_{\mathrm{h}}$ & $0.00(0.00)$ & $0.01(0.01)$ \\
\hline
\end{tabular}

Note. Model: $\mathrm{y}_{\mathrm{it}}=\eta_{\mathrm{oi}}+\eta_{1 \mathrm{i}} \mathrm{a}_{\mathrm{t}}+\eta_{2 \mathrm{i}} \mathrm{a}_{\mathrm{t}}^{2}+\varepsilon_{\mathrm{it}} \quad \mathrm{a}_{\mathrm{t}}=0,0.5,1.5,2.5,3.5,4.5$

$$
\begin{aligned}
& \eta_{0 \mathrm{i}}=\alpha_{0 \mathrm{k}}+\gamma_{0 \mathrm{k}} \mathrm{C}_{\mathrm{i}}+\zeta_{0 \mathrm{i}} \quad \eta_{1 \mathrm{i}}=\alpha_{1 \mathrm{k}}+\gamma_{1 \mathrm{k}} \mathrm{C}_{\mathrm{i}}+\zeta_{1 \mathrm{i}} \quad \eta_{2 \mathrm{i}}=\alpha_{2 \mathrm{k}}+\gamma_{2 \mathrm{k}} \mathrm{C}_{\mathrm{i}}+\zeta_{2 \mathrm{i}} \\
& \mathrm{C}_{\mathrm{i}}=\text { Concentration problems, Peer rejection, Reading, Lunch status, Race } \\
& \mathrm{V}(\zeta \mid \text { class } \mathrm{k})=\psi_{\mathrm{k}} \quad \mathrm{V}(\varepsilon \mid \text { class } \mathrm{k})=\Theta_{\mathrm{k}}
\end{aligned}
$$

Values with the same subscripts are significantly different from each other. CHAD = chronic high aggression-disruption; LMAD $=$ low moderate aggression-disruption; IAD = increasing aggression-disruption; LAD = low aggression-disruption; ASPD $=$ antisocial personality disorder.

partially known (using the Knownclass option of MPlus Version 3.12) and fully informed by the gender-specific growth mixture models. By partially known, we mean that, for example, girls could be in only those classes derived from the growth mixture models carried out for girls, but the exact class membership (CHAD, LMAD-IAD, or LAD) was reestimated within the multiple group model. Thus, the multiple group analysis resulted in a six-class model containing three classes for girls and three classes for boys. Parameter constraints from the separate gender models were included. This minimally constrained model is hereinafter referred to as the multiple group base model. Parameter estimates from the base model are presented in Table 1. These parameters are identical to those estimated for the gender-specific growth models.

The analyses were conducted in two steps. First, gender differences in class-specific initial levels and developmental course of aggressive-disruptive behaviors were examined. Once differences in growth parameters were determined, the second step explored gender differences in the class-specific prevalence of the distal outcomes of ASPD, arrest for a violent offense, and incarceration. The effects of covariates on class membership and growth parameters were allowed to vary by gender, given preliminary testing that revealed significant gender differences in these effects (see Table 1 for gender-specific estimates).

Initial levels and developmental course of aggressiondisruption. To test for gender differences on growth parameters (i.e., intercept level of aggression-disruption and growth over time) across analogous classes, we examined a series of nested models. As shown in Table 2, each of these nested models constrained a single parameter (e.g., intercept) to be equal for girls and boys in classes of interest. We used a log-likelihood ratio test to 


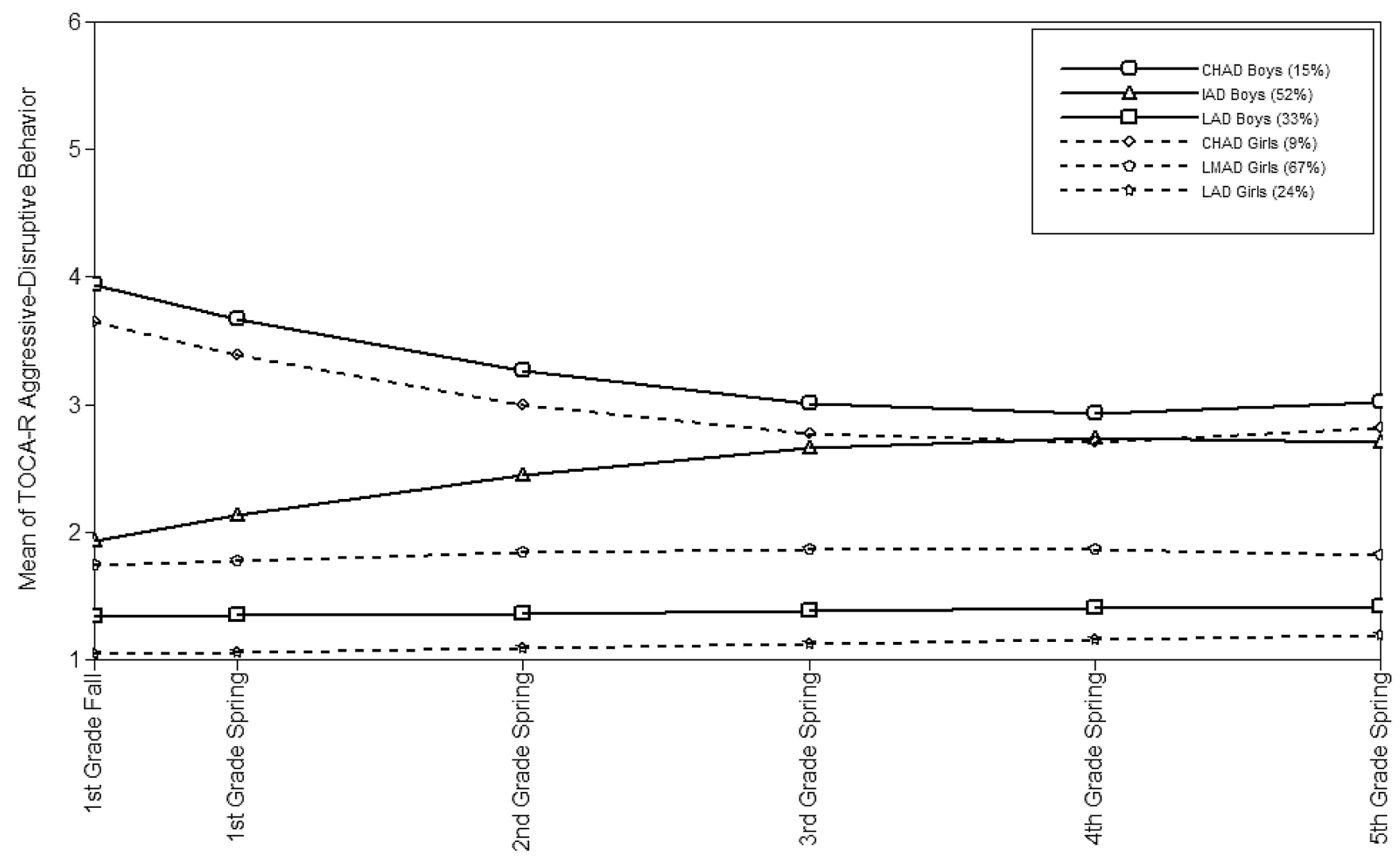

Grade and Semester

Figure 1. Final optimized multiple group model (girls and boys) of trajectories of aggressive-disruptive behavior over time, adjusted for the effects of covariates (race, free-lunch status, concentration problems, peer rejection, and reading achievement). TOCA-R = Teacher Observation of Classroom Adaptation-Revised; $\mathrm{CHAD}=$ chronic high aggression-disruption; IAD $=$ increasing aggression-disruption; LAD $=$ low aggression-disruption; LMAD = low moderate aggression-disruption. Percentages in legend are gender specific (i.e., sum to $100 \%$ for girls and boys separately).

compare each nested model with the base model (i.e., with the model that assumed the parameter to be unequal across genders). If the constraint did not result in a significantly worse fit over the base model, the parameter was considered to be equal for both genders. After each parameter of interest had been tested individually, all constraints that did not result in significantly worse model fit were tested simultaneously against the base model (i.e., omnibus tests). This sequence was repeated first examining differences in intercept (Models 1-4), then linear growth (Models 5-7), and then quadratic growth (Models 8-10).

On the basis of these analyses, the intercept, linear growth, and quadratic growth parameters for CHAD youth were found to be equivalent for girls and boys. Among LMAD-IAD youth, girls and boys had equivalent intercept values, but LMAD girls showed a significantly smaller increase in linear and quadratic growth than did IAD boys. Among LAD youth, girls started at a significantly lower level of aggressive-disruptive behavior than did boys (see Tables 1 and 2).

Distal outcomes. Distal outcome prevalences were tested in an identical fashion (Models 11-21; see Table 2). Among CHAD youth, girls and boys had equivalent rates of ASPD diagnosis and arrest for a violent offense, but girls had significantly lower rates of incarceration than did boys. Among LMAD-IAD youth, girls had significantly lower rates of ASPD, arrest for a violent offense, and incarceration than did boys. Among LAD youth, girls and boys had equivalent rates of violent arrests and incarceration, but boys were more likely to have ASPD.

We conducted post hoc tests to explore whether the lower rate of incarceration among CHAD girls, compared with CHAD boys, despite similar rates of arrest for violent offenses, could be explained by differences in rates of nonviolent arrests. A new variable, nonviolent arrest, was created from adult criminal records This distal outcome was substituted for violent arrest in the omnibus model containing all constraints on growth parameters (Model 10) and was explored for gender differences. Nested model testing suggested that the rate of nonviolent arrest for CHAD girls (18\%) was not significantly different from that of CHAD boys $(30 \%), \chi^{2}(1, N=150)=2.38, p=.123$. LMAD girls were significantly less likely than IAD boys to have a nonviolent arrest $(4 \%$ vs. $32 \%), \chi^{2}(1, N=434)=116.84, p<.001$; similarly, LAD girls were less likely than LAD boys to be arrested for a nonviolent offense ( $2 \%$ vs. $10 \%), \chi^{2}(1, N=352)=6.70, p<.01$. 
Table 2

Equivalence Between Girls and Boys on Growth Parameters and Distal Outcome Prevalences Using Multiple Group Analysis

\begin{tabular}{|c|c|c|c|c|c|c|}
\hline \multirow[b]{2}{*}{ Type of model } & \multicolumn{2}{|c|}{ Model fit } & \multirow{2}{*}{$\begin{array}{c}\text { Model } \\
\text { comparison }\end{array}$} & \multicolumn{3}{|c|}{$\begin{array}{l}\text { Likelihood ratio } \\
\text { test }^{\mathrm{a}}\end{array}$} \\
\hline & Log-likelihood & No. free & & $\chi^{2}$ diff. & $d f$ & $p$ \\
\hline $\mathrm{H}_{0}$ : Base model (no constraints across classes) & -6667.00 & 107 & & & & \\
\hline \multicolumn{7}{|l|}{ Equivalence of intercept parameters } \\
\hline 1. Constrain LAD-LAD intercepts & -6680.85 & 106 & 1 vs. $\mathrm{H}_{0}$ & 27.70 & 1 & .000 \\
\hline 2. Constrain LMAD-IAD intercepts & -6667.02 & 106 & 2 vs. $\mathrm{H}_{0}$ & 0.04 & 1 & .842 \\
\hline 3. Constrain CHAD-CHAD intercepts & -6667.93 & 106 & 3 vs. $\mathrm{H}_{0}$ & 1.86 & 1 & .173 \\
\hline 4. Omnibus test of all (i.e., Models 2 and 3 ) nonsignificant intercept constraints & -6668.28 & 105 & 4 vs. $\mathrm{H}_{0}$ & 2.56 & 2 & .278 \\
\hline \multicolumn{7}{|l|}{ Equivalence of linear growth parameters ${ }^{\mathrm{b}}$} \\
\hline 5. Model $4+$ constrain LMAD-IAD linear growth & -6685.25 & 104 & 5 vs. 4 & 33.94 & 1 & .000 \\
\hline 6. Model $4+$ constrain CHAD-CHAD linear growth & -6668.87 & 104 & 6 vs. 4 & 1.18 & 1 & .277 \\
\hline 7. Omnibus test of nonsignificant linear parameters (i.e., Model $4+$ Model 6) & -6668.87 & 104 & 7 vs. 4 & 1.18 & 1 & .277 \\
\hline \multicolumn{7}{|l|}{ Equivalence of quadratic growth parameters ${ }^{\mathrm{c}}$} \\
\hline 8. Model $7+$ constrain LMAD-IAD quadratic growth & -6675.28 & 103 & 8 vs. 7 & 12.82 & 1 & .000 \\
\hline 9. Model $7+$ constrain CHAD-CHAD quadratic growth & -6669.08 & 103 & 9 vs. 7 & 0.42 & 1 & .517 \\
\hline 10. Omnibus test of nonsignificant quadratic growth parameters (Model $7+$ Model 9) & -6669.08 & 103 & 10 vs. 7 & 0.42 & 1 & .517 \\
\hline \multicolumn{7}{|l|}{ Equivalence of distal outcome prevalences } \\
\hline 11. Model $10+$ constrain LAD-LAD ASPD prevalences & -6671.12 & 102 & 11 vs. 10 & 4.08 & 1 & .043 \\
\hline 12. Model $10+$ constrain LAD-LAD violent offense prevalences & -6669.82 & 102 & 12 vs. 10 & 1.48 & 1 & .224 \\
\hline 13. Model $10+$ constrain LAD-LAD incarceration prevalences & -6670.59 & 102 & 13 vs. 10 & 3.02 & 1 & .082 \\
\hline 14. Model $10+$ constrain LMAD-IAD ASPD prevalences & -6689.10 & 102 & 14 vs. 10 & 40.04 & 1 & .000 \\
\hline 15. Model $10+$ constrain LMAD-IAD violent offense prevalences & -6723.20 & 102 & 15 vs. 10 & 108.24 & 1 & .000 \\
\hline 16. Model $10+$ constrain LMAD-IAD incarceration prevalences & -6675.17 & 102 & 16 vs. 10 & 12.18 & 1 & .000 \\
\hline 17. Model $10+$ constrain CHAD-CHAD ASPD prevalences & -6669.89 & 102 & 17 vs. 10 & 1.62 & 1 & .203 \\
\hline 18. Model $10+$ constrain CHAD-CHAD violent offense prevalences & -6676.01 & 102 & 18 vs. 10 & 13.86 & 1 & .000 \\
\hline 19. Model $10+$ constrain CHAD-CHAD incarceration prevalences & -6669.09 & 102 & 19 vs. 10 & 0.02 & 1 & .888 \\
\hline 20. Omnibus test of all nonsignificant constraints on distal outcome prevalences & -6672.05 & 99 & 20 vs. 10 & 5.94 & 4 & .204 \\
\hline 21. Omnibus test of all nonsignificant constraints & -6672.05 & 99 & 21 vs. $\mathrm{H}_{0}$ & 10.10 & 8 & .258 \\
\hline
\end{tabular}

Note. $\quad \mathrm{LAD}=$ low aggression-disruption; $\mathrm{LMAD}=$ low moderate aggression-disruption; $\mathrm{IAD}=$ increasing aggression-disruption; $\mathrm{CHAD}=$ chronic high aggression-disruption; ASPD = antisocial personality disorder.

${ }^{a}$ The formula for the chi-square difference log-likelihood ratio test is -2 (Model $\times \log$-likelihood - Model Y log-likelihood). ${ }^{\mathrm{b}}$ The equivalence of linear growth parameters for the LAD group was not tested because the parameters were set to zero. ${ }^{\mathrm{c}}$ The equivalence of quadratic growth parameters for the LAD group was not tested because the parameters were set to zero.

\section{Discussion}

The present study addressed gaps in the literature regarding gender differences in trajectories of early aggressive-disruptive behavior and their associations to later antisocial outcomes. We examined individual differences in the development of girls' aggressive-disruptive behavior from school entry through middle childhood and whether trajectory classifications were associated with distal antisocial outcomes in young adulthood. We then tested for gender differences in the level and course of aggressive-disruptive behavior trajectories and the differential association between growth trajectories and later adult antisocial behavior.

\section{Aggressive-Disruptive Behavior Trajectories and Later Antisocial Outcomes}

Applying growth mixture modeling to the development of aggressive-disruptive behavior in girls revealed three distinct trajectories. We found evidence for one early-starter pathway, the CHAD group ( $9 \%$ in the present study), who displayed consistently high levels of aggression-disruption. Consistent with Bierman et al. (2004), it is possible that our inclusion of a broadband measure of early antisocial behavior, which included both aggres- sive and disruptive behaviors, allowed us to detect a distinct early-starter pathway with a relatively high prevalence rate. Indeed, the percentage of girls with the early-starter pattern in the present study was higher than that found in a previous study $(2.1 \%$ of girls; Fergusson \& Horwood, 2002) that used a more serious definition of antisocial behavior (i.e., parent- and self-reported offenses) to define trajectories. As predicted by the Moffitt (1993) and Patterson et al. (1989) models, girls with CHAD in the present study went on to have the highest rates of antisocial behavior in young adulthood.

The majority of girls (67\%) fell into the second trajectory identified in this study, the LMAD trajectory. Although other modeling studies have found trajectories of girls with moderate levels of aggression (Broidy et al., 2003) or aggression-disruption (Cote et al., 2001) during elementary school, the proportion of girls with this pattern (and with the CHAD pattern) also was larger in the present study than in previous work. One potential explanation for this finding is that, unlike previous work (which used samples primarily from Canada and New Zealand), the present study examined trajectories among girls living in urban neighborhoods in the United States characterized by high levels of violent crime and disorganization. It may be that aggressive-disruptive behavior among girls is more normative in these communities and thus is 
more likely to be accepted or tolerated by peers and adults (Ingoldsby \& Shaw, 2002).

In other studies of girls' early antisocial behavior, results have been mixed with regard to whether girls with moderate aggression-disruption are at risk for antisocial outcomes. Cote et al. (2001) found that girls with moderate levels of disruptive behavior had higher rates of conduct problems but were not at significantly increased risk for a diagnosis of conduct disorder. Similarly, in the present study, LMAD girls were not at increased risk for incarceration or ASPD but were more likely than nonaggressive-nondisruptive girls to be arrested for a violent offense. Taken together, a plausible hypothesis is that girls displaying this pattern are particularly vulnerable to stressors later in development (e.g., early puberty, romantic involvement with deviant males; Graber, Lewinsohn, Seeley, \& Brooks, 1997; ZahnWaxler \& Polanichka, 2004) and will experience some antisocial outcomes if such stressors are encountered. Research that explores gender-specific and nonspecific risk factors among girls with LMAD and with CHAD is needed.

\section{Gender Differences in Trajectories and Antisocial Outcomes}

To our knowledge, the present study is the first to explicitly test for gender differences in early (from school entry through fifth grade) aggressive-disruptive behavior trajectories within the growth mixture framework. A few gender differences were found. Among youth with LAD, girls had slightly lower initial levels of aggressive-disruptive behavior (i.e., intercept) than did boys. In addition, we found a class of increasing aggressive-disruptive behavior over time among boys but not among girls.

Surprisingly, among youth with CHAD trajectories, girls' and boys' initial levels and growth in aggression-disruption over time (i.e., intercepts and slopes) were identical. This finding runs counter to the results of Broidy et al. (2003), who suggested (but did not explicitly test) that, across four samples of boys and girls, girls classified to a chronic high trajectory class had lower levels of physical aggression than their male counterparts classified to that trajectory. Thus, the present study is the first to identify a group of girls whose levels of chronic high aggressive-disruptive behavior on entry to elementary school are as high as levels found for boys. Again, one potential explanation for this finding is that the present study examines trajectories among youth living in urban neighborhoods in the United States. Another possibility is that the inclusion of disruptive behaviors along with aggression in the identification of trajectories results in greater similarities between girls and boys. Replication of these results in similar communities along with the use of a comparable measure of antisocial behavior is essential to ensure that this finding is not an artifact of our sample.

In terms of trajectory classes predicting distal outcomes, we found several similarities and differences between genders. One similarity was that, among CHAD youth, girls and boys had equivalent rates of ASPD, arrest for a violent offense, and arrest for a nonviolent offense. These findings are inconsistent with the results of Fergusson and Horwood (2002) who suggested (but again did not explicitly test) that rates of offending were lower among girls with the early-starter pattern than among boys with the same pattern. Although differences in population (i.e., U.S. urban, economically disadvantaged youth of primarily African descent vs. nonurban, New Zealand youth of primarily European descent) and measurement (i.e., aggression-disruption vs. offending) may explain this apparent difference between the studies, these discrepancies highlight the need for continued empirical testing of assumed gender differences in future research.

In terms of gender differences, girls with CHAD trajectories had significantly lower rates of incarceration than CHAD boys. At least two interpretations of this finding are possible. First, it may be that, although many girls with CHAD trajectories engage in violent and nonviolent criminal acts in young adulthood, their offenses are less severe than those of boys and thus less likely to result in incarceration. The data available in the present study did not allow for the assessment of offense severity beyond crude categorization. Future research should examine the role of offense severity outside the categorical distinctions (i.e., violent and nonviolent) used here. A second (but not mutually exclusive) explanation is that, although girls and boys with CHAD trajectories commit crimes of similar severity, girls are less likely to be incarcerated than boys because of gender biases in sentencing (Armstrong, 1999; Payne, Gainey, Triplett, \& Danner, 2004).

Empirical comparisons between girls in the LMAD trajectory and boys in the IAD trajectory confirmed that these groups are not equivalent. In addition to their differences in growth in aggressivedisruptive behavior over time, boys with an IAD trajectory are at much higher risk for ASPD and incarceration than are girls with an LMAD trajectory. Thus, the present study suggests that there is only one early aggression-disruption trajectory (CHAD) that consistently places girls at high risk for antisocial outcomes, whereas for boys there are two (CHAD and IAD). However, it would be wrong to assume that girls with LMAD trajectories are doing well (Bierman et al., 2004). Indeed, in the present study, LMAD girls were at some increased risk for violent arrest relative to LAD girls, and other research has suggested that girls with moderate levels of disruptive behavior are at risk for a range of negative outcomes such as depression, school dropout, and early pregnancy (Loeber \& Keenan, 1994; Zoccolillo, Paquette, Azar, Cote, \& Tremblay, 2004).

\section{Limitations and Implications for Prevention}

In the present study, we did not examine other forms of antisocial behavior (e.g., relational aggression) that may be especially significant for girls. Cross-sectional and short-term longitudinal research has found that relational aggression is associated with peer rejection and depression during childhood (Crick, 1996; Crick \& Grotpeter, 1995), but a link to serious antisocial behavior problems later in life has yet to be established. Moreover, relational and physical aggression tends to be highly correlated among both girls and boys (Henington, Hughes, Cavell, \& Thompson, 1998; Tomada \& Schneider, 1997), suggesting that girls who display high rates of physical aggression also may exhibit high rates of relational aggression. More longitudinal research that examines disruptive behavior, physical aggression, and relational aggression across the life span and explores their association to antisocial outcomes in young adulthood is necessary to understand the unique and common effects of these different forms of antisocial behavior for girls. 
Given the constraints of the variables available in our data set, we were unable to control for the effects of risk factors that may be particularly important to the development of girls' antisocial behavior such as a history of trauma or early pubertal status (Graber et al., 1997). Of the covariates that we did examine, our measure of peer rejection was limited to a single item, which may not have captured subtleties in peer-relation characteristics such as peer neglect. In addition, ratings of aggressive-disruptive behavior, concentration problems, and peer rejection in first grade were based solely on teacher reports, raising possible concerns about spurious results due to shared method variance. However, mitigating this concern is the fact that ratings of aggressive-disruptive behavior made after first grade were made by different teachers. Similarly, later outcomes were collected from state agencies, as well as from the youth themselves. Another limitation of our data set was that our measurement of aggressive-disruptive behavior was limited to the elementary school years; consequently, we were not able to detect what Patterson et al. (1989) referred to as a "desister" group - that is, those youth whose initially high levels of aggressive-disruptive behavior declines to normal levels by adolescence.

To our knowledge, the present study is the first to identify pathways of antisocial behavior development within an epidemiological sample of urban, primarily African American girls and, as such, fills an important gap in the literature, which to date has focused primarily on boys and on youth of European American descent (Broidy et al., 2003; Cote et al., 2001). In the United States, African American youth are among the most socioeconomically disadvantaged and, consequently, may experience a wide array of risk factors for criminal behavior (Miller-Johnson, Moore, Underwood, \& Coie, 2005). African American girls, in particular, are grossly overrepresented in the juvenile justice system (Federal Bureau of Investigation, 2003). The present findings suggest that African American youth exhibit aggressive-disruptive behavior trajectories that are similar to those seen in European American youth. Moreover, as with European Americans, these trajectories are associated differentially with serious forms of antisocial behavior in young adulthood. Research that examines specific risk and protective mechanisms for antisocial behavior among African American youth is needed.

Overall, our findings suggest that early aggressive-disruptive behavior is a significant problem for some girls and is a worthy target for early identification and intervention efforts. We found a trajectory class of girls who, like their male counterparts, engaged in chronically high levels of antisocial behavior in childhood and who were at increased risk for antisocial outcomes in adolescence and young adulthood. Girls' first-grade levels of overt aggressivedisruptive behavior are likely to be indicative of the course they follow during the elementary school years and the likelihood for continued, more serious involvement in antisocial behavior in young adulthood. Accordingly, teacher ratings of antisocial behavior as early as first grade could be used to identify girls in need of preventive intervention. However, in light of the limitations of a single assessment time point (see Petras et al., 2004, 2005), multiple assessment time points may be needed to improve accuracy in selecting children for preventive interventions. Muthén et al. (2002) have reported on the use of growth mixture modeling for ascertaining selection accuracy as a function of the number of assessment time points. Such a method may prove helpful to prevention scientists in determining the fewest number of time points necessary to identify girls for preventive intervention efforts.

\section{References}

American Psychiatric Association. (1994). Diagnostic and statistical manual of mental disorders (4th ed.). Washington, DC: Author.

Arbuckle, J. L. (1996). Full information estimation in the presence of incomplete data. In G. A. Marcoulides \& R. E. Schumacker (Eds.), Advanced structural equation modeling: Issues and techniques (pp. 243-277). Mahwah, NJ: Erlbaum.

Armstrong, I. (1999). Women and their "uncontrollable impulses": The medicalization of women's crime and differential gender sentencing. Psychiatry, Psychology, and the Law, 6, 67-77.

Bauer, D. J., \& Curran, P. J. (2003). Distributional assumptions of growth mixture models: Implications for overextraction of latent trajectory classes. Psychological Methods, 8, 338-363.

Bierman, K. L., Bruschi, C., Domitrovich, C., Fang, G. Y., Miller-Johnson, S., \& the Conduct Problems Prevention Research Group. (2004). Early disruptive behaviors associated with emerging antisocial behavior among girls. In M. Putallaz \& K. L. Bierman (Eds.), Aggression, antisocial behavior, and violence among girls (pp. 137-161). New York: Guilford Press.

Broidy, L. M., Nagin, D. S., Tremblay, R. E., Bates, J. E., Brame, B., Dodge, K. A., et al. (2003). Developmental trajectories of childhood disruptive behaviors and adolescent delinquency: A six-site, crossnational study. Developmental Psychology, 39, 222-245.

Cote, S., Zoccolillo, M., Tremblay, R. E., Nagin, D., \& Vitaro, F. (2001). Predicting girls' conduct disorder in adolescence from childhood trajectories of disruptive behaviors. Journal of the American Academy of Child and Adolescent Psychiatry, 40, 678-684.

Crick, N. R. (1996). The role of overt aggression, relational aggression, and prosocial behavior in the prediction of children's future social adjustment. Child Development, 67, 2317-2327.

Crick, N. R., \& Grotpeter, J. K. (1995). Relational aggression, gender, and social-psychological adjustment. Child Development, 66, 710-722.

Dolan, L. J., Kellam, S. G., Brown, C. H., Werthamer-Larsson, L., Rebok, G. W., Mayer, L. S., et al. (1993). The short-term impact of two classroom-based preventive interventions on aggressive and shy behaviors and poor achievement. Journal of Applied Developmental Psychology, 14, 317-345.

Ensminger, M. E., Forrest, C. B., Riley, A. W., Kang, M., Green, B. F., Starfield, B., \& Ryan, S. A. (2000). The validity of measures of socioeconomic status of adolescents. Journal of Adolescent Research, 15, 392-419.

Federal Bureau of Investigation. (2003). Uniform crime reports. Washington, DC: U.S. Government Printing Office.

Fergusson, D. M., \& Horwood, L. J. (2002). Male and female offending trajectories. Development and Psychopathology, 14, 159-177.

Graber, J. A., Lewinsohn, P. M., Seeley, J. R., \& Brooks, J. (1997). Is psychopathology associated with the timing of pubertal development? Journal of the American Academy of Child and Adolescent Psychiatry, 36, 1768-1776.

Greenbaum, P. E., Brown, E. C., Brown, C. H., Kellam, S. G., Rolande, M., \& Poduska, J. M. (2000, June). Invariance of factor structure and loadings of the TOCA-R: Gender differences in aggressive behavior from first grade into middle school. Poster presented at the 8th Annual Meeting of the Society for Prevention Research, Montreal, Quebec, Canada.

Henington, C., Hughes, J. N., Cavell, T. A., \& Thompson, B. (1998). The role of relational aggression in identifying aggressive boys and girls. Journal of School Psychology, 36, 457-477.

Ingoldsby, E. M., \& Shaw, D. S. (2002). Neighborhood contextual factors 
and early-starting antisocial pathways. Clinical Child and Family Psychology Review, 5, 21-55.

Jöreskog, K. G., \& Goldberger, A. S. (1975). Estimation of a model with multiple indicators and multiple causes of a single latent variable. Journal of the American Statistical Association, 70, 631-639.

Keenan, K., \& Shaw, D. (1997). Developmental and social influences on young girls' early problem behavior. Psychological Bulletin, 121, 95113.

Kobak, K. A., Taylor, L. H., Dottl, S. L., Greist, J. H., Jefferson, J. W., Burroughs, D., et al. (1997). A computer-administered telephone interview to identify mental disorders. Journal of the American Medical Association, 278, 905-910.

Little, R. J. (1995). Modeling the dropout mechanism in repeated-measures studies. Journal of the American Statistical Association, 90, 1112-1121.

Loeber, R., \& Keenan, K. (1994). Interaction between conduct disorder and its comorbid conditions: Effects of age and gender. Clinical Psychology Review, 14, 497-523.

Miller-Johnson, S., Moore, B. L., Underwood, M. K., \& Coie, J. D. (2005). African-American girls and physical aggression: Does stability of childhood aggression predict later negative outcomes? In D. J. Pepler, K. C. Masten, C. Webster, \& K. S. Levene (Eds.), The development and treatment of girlhood aggression (pp. 75-95). Mahwah, NJ: Erlbaum.

Moffitt, T. E. (1993). Adolescent-limited and life-course persistent antisocial behavior: A developmental taxonomy. Psychological Review, 100, 674-701.

Moffitt, T. E., Caspi, A., Rutter, M., \& Silva, P. A. (2001). Sex differences in antisocial behavior: Conduct disorder, delinquency, and violence in the Dunedin longitudinal study. Cambridge, United Kingdom: Cambridge University Press.

Muthén, B. (2004). Latent variable analysis: Growth mixture modeling and related techniques for longitudinal data. In D. Kaplan (Ed.), Handbook of quantitative methodology for the social sciences (pp. 345-368). Newbury Park, CA: Sage.

Muthén, B., Brown, C. H., Masyn, K., Jo, B., Khoo, S. T., Yang, C. C., et al. (2002). General growth mixture modeling for randomized preventive interventions. Biostatistics, 3, 459-475.

Muthén, B., \& Muthén, L. K. (2004). Mplus users guide. Los Angeles: Author.

Muthén, B., \& Shedden, K. (1999). Finite mixture modeling with mixture outcomes using the EM algorithm. Biometrics, 6, 463-469.

Nagin, D., \& Tremblay, R. E. (1999). Trajectories of boys' physical aggression, opposition, and hyperactivity on the path to physically violent and nonviolent juvenile delinquency. Child Development, 70, $1181-1196$

Olsen, M. K., \& Schaefer, J. L. (2001). A two-part random effects model for semicontinuous longitudinal data. Journal of the American Statistical Association, 96, 730-745.

Patterson, G. R., DeBaryshe, B. D., \& Ramsey, E. (1989). A developmental perspective on antisocial behavior. American Psychologist, 44, 329335 .

Payne, B. K., Gainey, R. R., Triplett, R. A., \& Danner, M. J. E. (2004). What drives punitive beliefs? Demographic characteristics and justifications for sentencing. Journal of Criminal Justice, 32, 195-206.

Petras, H., Ialongo, N., Lambert, S. F., Barrueco, S., Schaeffer, C., Chilcoat, H., \& Kellam, S. (2005). The utility of teacher ratings of aggression during the elementary school years in identifying later violence in adolescent females-A replication. Journal of the American Academy of Child and Adolescent Psychiatry, 16, 790-797.
Petras, H., Schaeffer, C., Ialongo, S., Muthén, B., Lambert, S., Poduska, J., \& Kellam, S. (2004). When the course of aggressive behavior in childhood does not predict antisocial outcomes in adolescence and adulthood: An examination of potential explanatory variables. Development and Psychopathology, 16, 919-941.

Rohde, P., Lewinsohn, P. M., \& Seeley, J. R. (1997). Comparability of telephone and face-to-face interviews in assessing Axis I and II disorders. American Journal of Psychiatry, 154, 1593-1598.

Schaeffer, C. M., Petras, H., Ialongo, N., Poduska, J., \& Kellam, S. (2003). Modeling growth in boys aggressive behavior across elementary school: Links to later criminal involvement, conduct disorder, and antisocial personality disorder. Developmental Psychology, 39, 1020-1035.

Schafer, J. L., \& Graham, J. W. (2002). Missing data: Our view of the state of the art. Psychological Methods, 7, 147-177.

Schwartz, G. (1978). Estimating the dimension of a model. The Annals of Statistics, 6, 461-464.

Shaw, D. S., Gilliom, M., Ingoldsby, E. M., \& Nagin, D. S. (2003). Trajectories leading to school-age conduct problems. Developmental Psychology, 39, 189-200.

Silverthorn, P., \& Frick, P. J. (1999). Developmental pathways to antisocial behavior: The delayed-onset pathway in girls. Development and Psychopathology, 11, 101-126.

Slutske, W. S., True, W. R., Scherrer, J. F., Goldberg, J., Bucholz, K. K., Heath, A. C., et al. (1998). Long-term reliability and validity of alcoholism diagnoses and symptoms in a large national telephone interview survey. Alcoholism: Clinical \& Experimental Research, 22, 553-558.

Tomada, G., \& Schneider, B. H. (1997). Relational aggression, gender, and peer acceptance: Invariance across culture, stability over time, and concordance among informants. Developmental Psychology, 33, 601609.

Turner, R. J., \& Gil, A. G. (2002). Psychiatric and substance disorders in South Florida: Racial/ethnic and gender contrasts in a young adult cohort. Archives of General Psychiatry, 59, 43-50.

Wardrop, J. L. (1989). Review of the California Achievement Tests, Forms E and F. In J. Close Conoley \& J. Kramer (Eds.), The tenth mental measurements yearbook (pp. 128-133). Lincoln: University of Nebraska Press.

Webster-Stratton, C. (1996). Early-onset conduct problems: Does gender make a difference? Journal of Consulting and Clinical Psychology, 64, $540-551$.

Werthamer-Larsson, L., Kellam, S. G., \& Wheeler, L. (1991). Effect of first-grade classroom environment on child shy behavior, aggressive behavior, and concentration problems. American Journal of Community Psychology, 19, 585-602.

Zahn-Waxler, C., \& Polanichka, N. (2004). All things interpersonal: Socialization and female aggression. In M. Putallaz \& K. L. Bierman (Eds.), Aggression, antisocial behavior, and violence among girls: A developmental perspective (pp. 48-68). New York: Guilford Press.

Zoccolillo, M., Paquette, D., Azar, R., Cote, S., \& Tremblay, R. (2004). Parenting as an important outcome of conduct disorder in girls. In M. Putallaz \& K. L. Bierman (Eds.), Aggression, antisocial behavior, and violence among girls: A developmental perspective (pp. 242-261). New York: Guilford Press.

Received December 28, 2004

Revision received October 22, 2005 Accepted January 5, 2006 